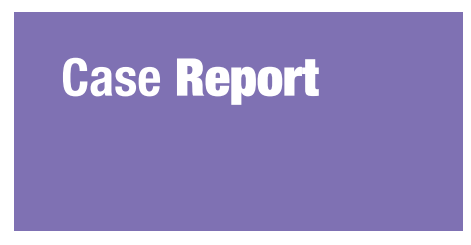

\title{
Embolisation of External Jugular Vein Aneurysm: A Case Report
}

Submitted: 2 Jun 2015

Accepted: 2 Aug 2016

Online: 29 Dec 2017
Arvin Rajadurai ${ }^{1}$, Aida Abdul Aziz ${ }^{1}$, Noor Aida Mat Daud ${ }^{1}$, Abdul Fatah Abdul Wahab ${ }^{2}$, Ahmad Sobri Muda ${ }^{3}$

1 Department of Radiology, Hospital Sungai Buloh, Selangor, Malaysia

2 Department of Otorhinolaryngology, Hospital Sungai Buloh, Selangor, Malaysia

3 Department of Radiology, Universiti Kebangsaan Malaysia Medical Centre, Kuala Lumpur, Malaysia

To cite this article: Rajadurai A, Abdul Aziz A, Mat Daud NA, Abdul Wahab AF, Muda AS. Embolisation of external jugular vein aneurysm: a case report. Malays J Med Sci. 2017;24(6):107-112. https://doi.org/10.21315/ mjms2017.24.6.14

To link to this article: https://doi.org/10.21315/mjms2017.24.6.14

\begin{abstract}
Introduction: Venous aneurysms are not as common as their arterial counterpart. The choice of management is debatable. Case Report: We present the case of a teenage boy with left external jugular vein aneurysm treated by endovascular technique. Embolisation was done using pushable microcoils with access via the femoral vein and direct puncture. Conclusion: This form of treatment provides a less-invasive option with a more concrete evaluation of the venous abnormality and its drainage during venous aneurysm occlusion.
\end{abstract}

Keywords: jugular vein aneurysm, embolisation, endovascular treatment, venous aneurysm

\section{Introduction}

Venous aneurysms were first described in 1928 and are relatively uncommon $(1,2)$. A venous aneurysm is an isolated saccular or fusiform dilatation of a vein that occurs because of congenital weakness of the vessel wall. A secondary type of the superficial venous system occurs as a result of trauma, inflammation, degenerative change in the vessel wall or increased pressure within the vascular system, including varicose veins $(3,4)$.

Jugular vein ectasia typically takes a benign course and rarely poses life-threatening complications, although patients may experience choking, bluish discoloration, thrombosis and discomfort. In most cases, treatments are undertaken for cosmetic reasons. Surgery is the mainstay of treatment, in which the dilated and redundant veins are ligated.

We present a case of left external jugular vein aneurysm requiring treatment for cosmetic reasons. We opted for the endovascular treatment technique, as embolisation provides a less-invasive option with a more concrete evaluation of the venous drainage prior to venous aneurysm occlusion. A review of the literature reveals no similar case using endovascular treatment. The imaging findings, strategies and techniques of endovascular treatment are also described.

\section{Case Presentation}

A 15-year-old boy with no known medical illness presented with a painless left neck swelling for a duration of one year. This swelling progressively increased in size and became more prominent when strained. He had no symptoms of stridor or shortness of breath. He also had no history of prior trauma to the neck. Physical examination revealed a soft, non-pulsating, fluctuant, well-defined left anterior triangle neck swelling measuring approximately $3 \mathrm{~cm} \times 2 \mathrm{~cm}$. 
This lesion was more prominent on valsalva manoeuvre. Auscultation revealed no bruit over the lesion. The rest of the physical examination was unremarkable.

Laboratory tests confirmed no abnormality, and contrast-enhanced CT scan indicated that it was not a highly vascular tumour. CT scan revealed a left external jugular vein ectasia measuring $3.1 \mathrm{~cm} \times 2.6 \mathrm{~cm} \times 1.4 \mathrm{~cm}$. The reconstructed $3 \mathrm{D}$ image clearly depicts venous aneurysm (Figure 1). Ultrasound of the neck with colour Doppler flow imaging further confirmed the findings of a left external jugular vein ectasia that expanded in size upon valsalva manoeuvre (Figure 2).

The patient requested treatment for cosmetic reason, and the ENT team referred the patient to us for endovascular option. Diagnostic angiogram and venogram were performed for mapping embolisation in the same setting. A $5 \mathrm{~F}$ sheath and a $4 \mathrm{~F}$ sheath were inserted into the right femoral vein and right femoral artery, respectively. Diagnostic run of the left common carotid artery confirmed the external jugular vein aneurysm. The sac of the external jugular vein aneurysm was cannulated directly via percutaneous puncture by using $19 \mathrm{G}$ branula under ultrasound guidance (Figure 3a, Figure 3b). Percutaneous access of a superficial vascular lesion is feasible and can aid in embolic agent delivery.

Diagnostic venography confirmed external jugular aneurysm draining into the left subclavian vein with no thrombosis (Figure 4). The feeder vessel was cannulated via the venous system and embolised transvenously using a Hilal microcoil (COOK, 0.018 in. system, $4 \mathrm{~mm} \times 30 \mathrm{~mm}$ ), whilst the abnormal sac was
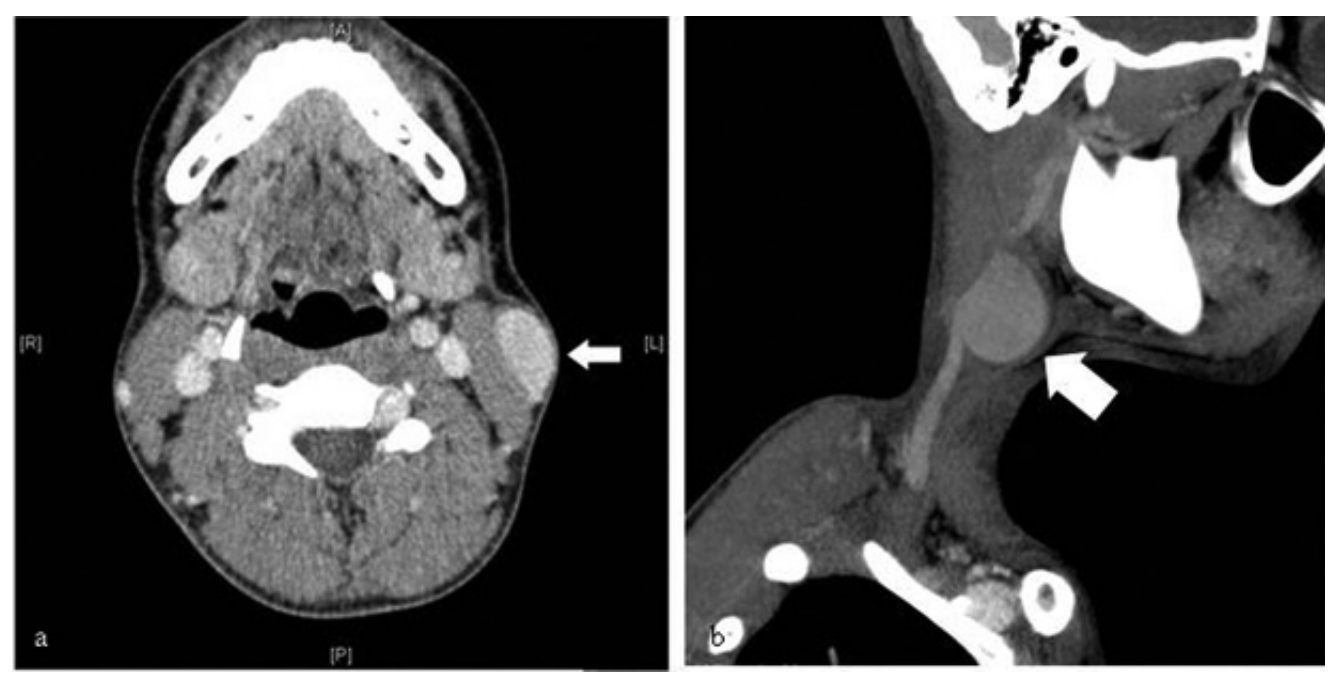

Figure 1. CT scan of the neck revealing a focal dilatation of the left external jugular vein. The reconstructed 3D image clearly depicts the venous aneurysm.
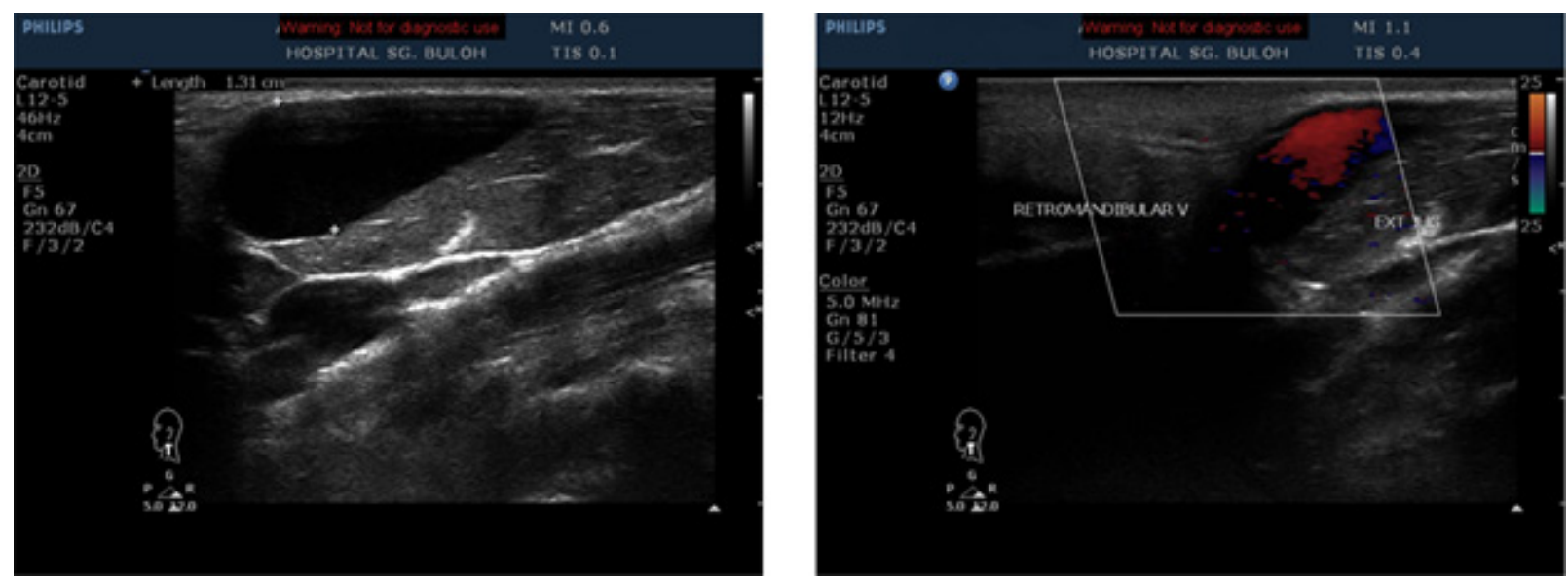

Figure 2. Duplex ultrasound of the neck demonstrating left external jugular vein aneurysm. 

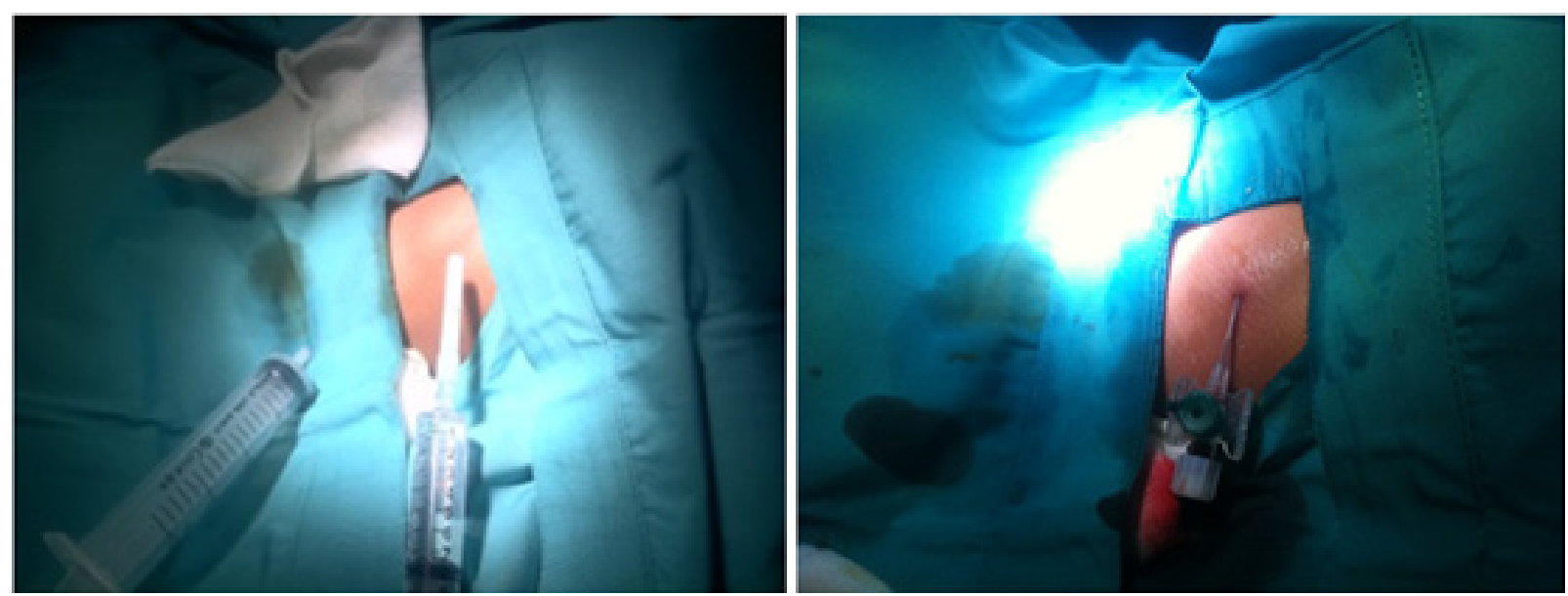

Figure 3a. Images on the table showing a dilated ectatic sac and direct puncture of the sac prior to left external jugular vein run.

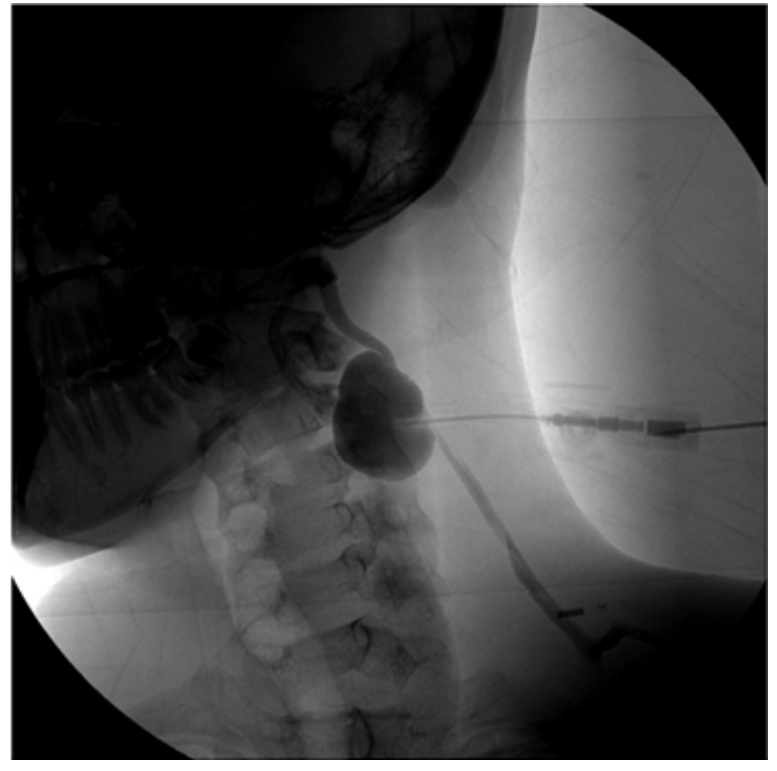

Figure 3b. Diagnostic run from a direct puncture of the external jugular venous ectatic sac.

embolised with two Nester embolisation coils (COOK, Nester fibred 0.018 in. system, 12-14 $\times$ $35 \mathrm{~mm}$ and $6-14 \times 35 \mathrm{~mm}$ ). There was complete occlusion of the ectatic left external jugular vein and the venous sac about 10 minutes after embolisation. The left internal jugular vein remained patent (Figure 5).

The patient was stable after the procedure and was discharged two days later with antibiotics. The lesion swelled marginally after one week but subsided after one month showing a small residual swelling with no increase in size on valsalva (Figure 6). No dragging sensation

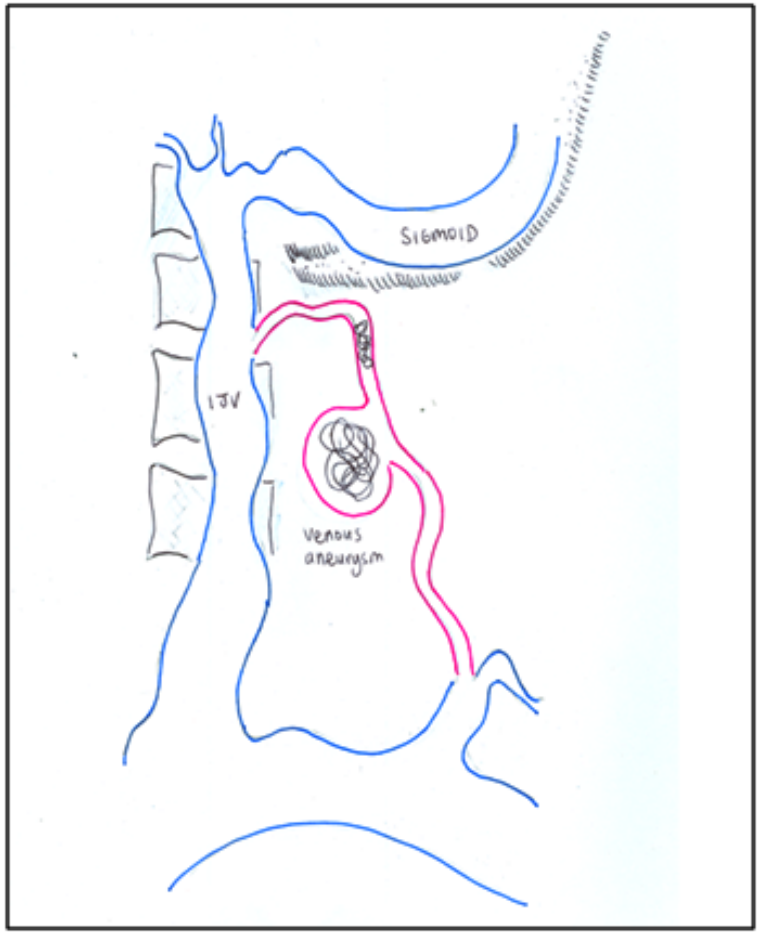

Figure 4. Diagramatic representation of veous aneurysm coiling.

or mass was observed in the neck. On followup, the coil was felt on the left side of the neck on palpation. The follow-up CT scan of the neck also revealed reduced flow through the ectatic segment with the coils noted in situ. The reconstructed $3 \mathrm{D}$ image illustrates the coil mass within the thrombosed venous aneurysm sac with some coils in the external jugular vein (Figure 7). 


\section{Discussion}

Few cases of external jugular venous aneurysm have been described in the literature. Typical differential diagnoses that are considered for a neck swelling with characteristic appearances on valsalva manoeuvre include laryngocele, branchial cyst, tumour or cysts of the upper mediastinum, cystic hygroma, thyroglossal cysts, dermoid cysts, cervical adenitis and metastatic adenopathy (5).

Case reports have shown that external jugular venous aneurysms commonly present as round masses that are mostly asymptomatic, unilateral, soft and non-pulsatile with no bruit on auscultation. Their distinguishing characteristics include enlargement with straining, crying, sneezing or valsalva manoeuvre, but they are difficult to isolate from internal jugular venous aneurysms by clinical assessment. The imaging modality of choice to establish the diagnosis is debatable; Candram et al. suggest that the use of Doppler ultrasonography is adequate. However, the use of multi-detector CT gives superior temporal and spatial resolution which provides images better demonstrating the vascular anomaly. In our case, we used CT, ultrasonography and colour Doppler flow imaging with venography performed prior to endovascular management.

Treatment is reserved for cosmetic reasons or when complications arise, such as thrombus formation, pulmonary embolism, spontaneous rupture and thrombophlebitis $(3,9-11)$. In our case, treatment was carried out for cosmetic reasons.

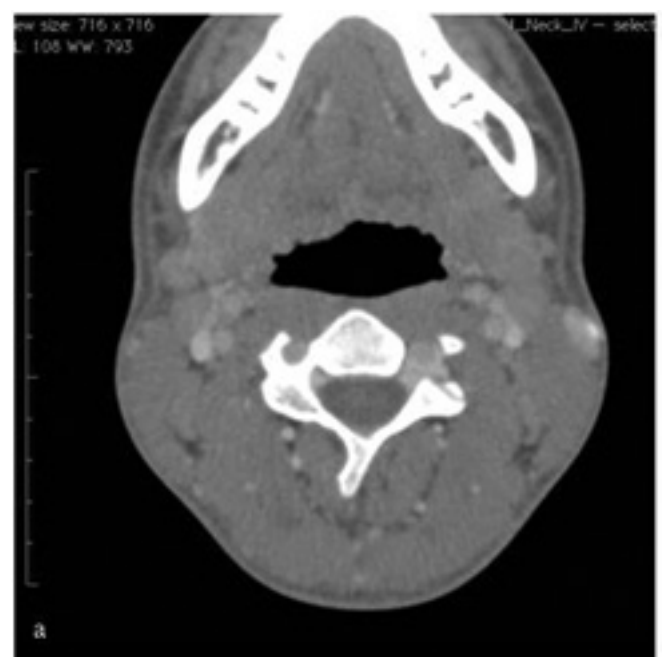

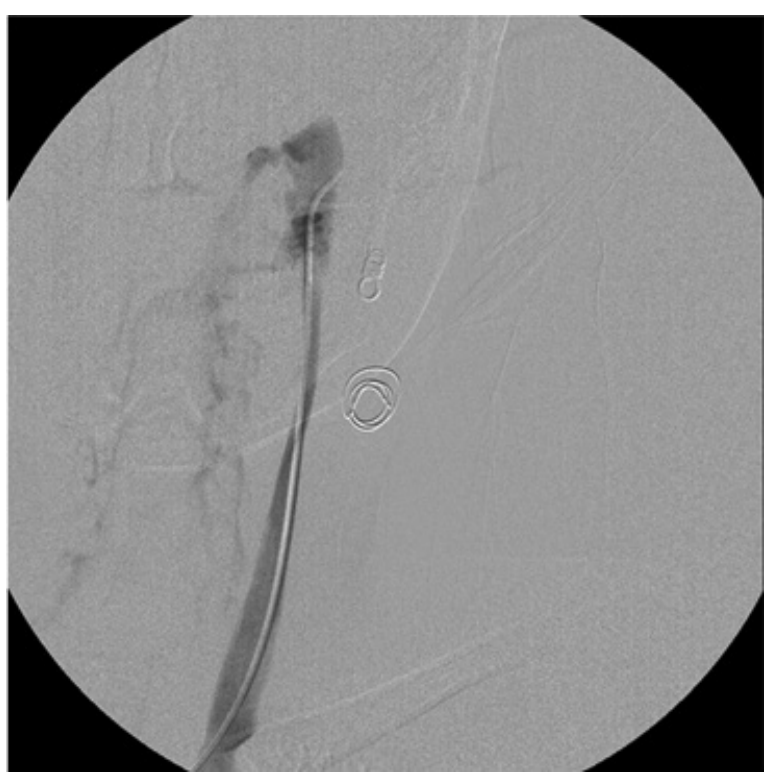

Figure 5. Post-coiling venogram demonstrating patent internal jugular vein. The ectatic sac was successfully embolised. One coil was in the external jugular venous aneurysm sac and another within the feeder.

Most studies share their surgical experience in the management of external jugular venous aneurysms. Potential complications that may arise from surgical intervention include complications from general anaesthesia, damage to local structures, surgical wound breakdown and infection, as well as haemorrhage. Surgical management can also lead to scar formation, and

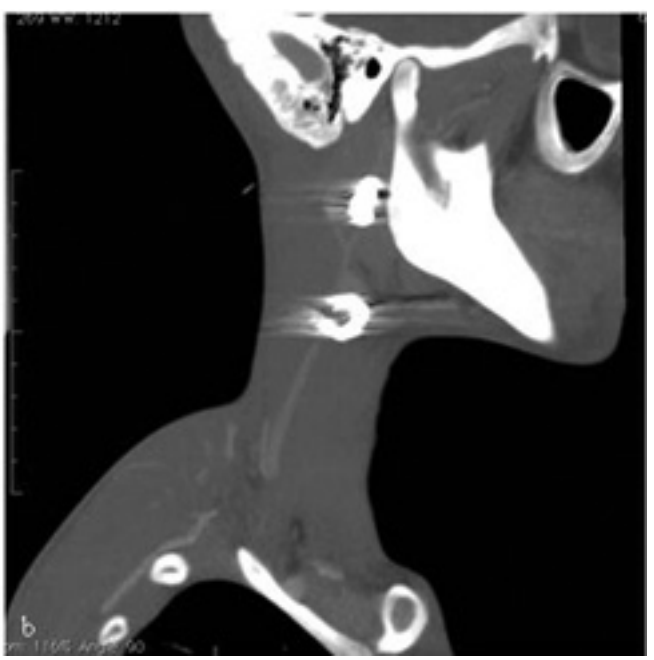

Figure 6. Follow-up after one month shows small residual swelling with no increase in size on valsalva. 


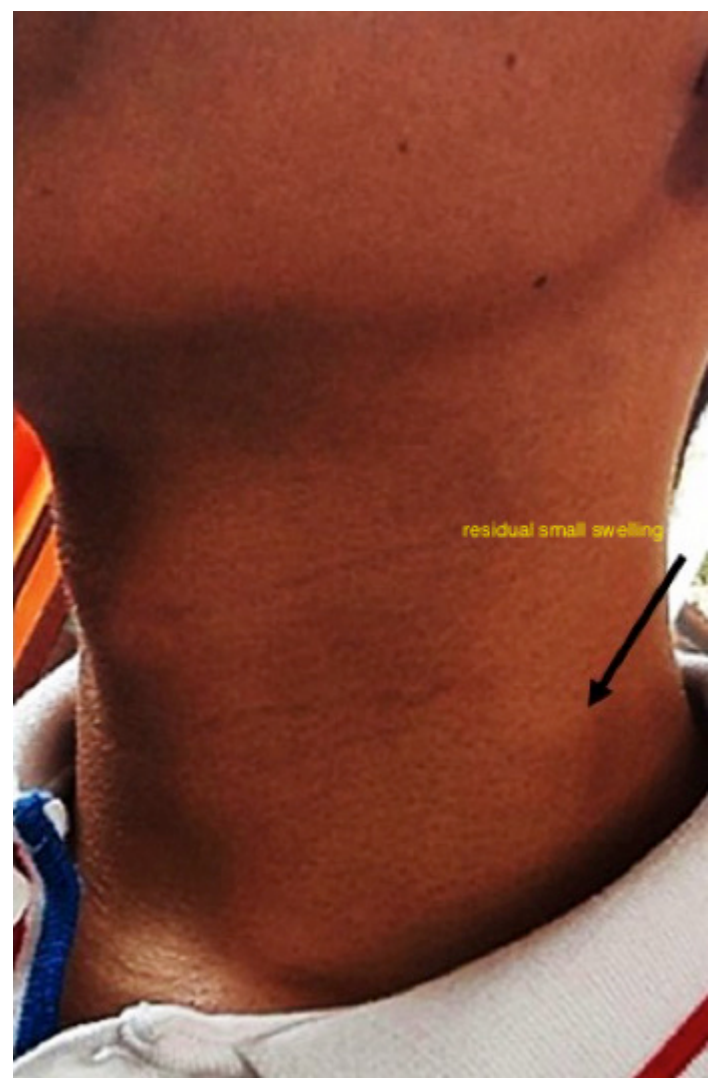

Figure 7. Follow-up CT scan demonstrating absent flow in the ectatic segment and normal flow within the external jugular vein. The reconstructed $3 \mathrm{D}$ image illustrates the coil mass within the thrombosed venous aneurysm sac with some coils in the external jugular vein.

for those with underlying hypertrophic scarring, it may be worse cosmetically than if they had not had the surgery at all.

Endovascular treatment of venous aneurysms is a minimally invasive management technique. Scarring is minimal compared with surgical management from femoral and local puncture sites. The need for general anaesthesia is overcome and provides treatment options for patients not suitable for general anaesthesia. Local injury is also comparably less from endovenous management than from open surgery.

In our case, we opted for embolisation, which is less invasive and with a comparable outcome for surgery management. Our patient was noted to have a marginal increase in the size of the lesion, postulated because of thrombosis of the venous sac that subsided completely within one month with no flow on Doppler and resolution on follow-up CT, indicating complete resolution.

\section{Conclusion}

External jugular venous aneurysms are uncommon. Treatment is either for cosmetic reasons or when related complications arise. Current literature supports conservative management or surgical excision for symptomatic patients. Endovascular treatment or embolisation serves as a minimally invasive option with potentially similar outcomes and cosmetic benefits as surgery.

\section{Acknowledgements}

The authors thank Ms Siti Farizwana for her technical help. They would also like to extend a big gratitude to the angiography team of Hospital Sg. Buloh for their cooperation.

\section{Authors' Contributions}

Conception and design: AR, ASM

Analysis and interpretation of the data: NAMD

Drafting of the article: AR, AAA, NAMD, AFAW, ASM

Critical revision of the article for important intellectual content: AAA, ASM

Final approval of the article: ASM

Provision of study materials or patients: ASM

Administrative, technical, or logistic support: AFAW

Collection and assembly of data: AR

\section{Correspondence}

Professor Dr Ahmad Sobri Muda MD (UKM), MMed (Rad) (USM)

Department of Radiology,

Universiti Kebangsaan Malaysia Medical Centre, Jalan Yaakob Latiff, 5600o Cheras,

Kuala Lumpur, Malaysia.

Tel: +6 o3 91458289

Fax: +6 0391737824

E-mail: sobri_muda@yahoo.com

\section{References}

1. Haris RI. Congenital venous cysts of the mediastinum. Ann Surg 1928; 88:953-956. https://doi.org/10.1097/00000658-19281100000015 
2. Paleri V, Gopalakrishnan S. Jugular phelebectasia: theory of pathogenesis and review of the literatüre. Int $J$ Pediatr. Otorhinolaryngol 2001;57:155-159. https://doi.org/10.1016/So1655876(00)00435-3

3. Bowdler DA, Singh SD. Internal Jugular phlebectasia. Int $J$ of Pediatr Otorhinolaryngol 1986;12:165-171. https://doi.org/10.1016/So165$5876(86) 80073-8$

4. Bergan JJ, Pascarella L, Sparks S, Mekenas L. Superficial venous aneurysm: prevalence and etiology. In: The 2003UIP World Congress Chapter Meeting manual. San Diego, CA: American College of Phlebology Foundation; 2003. 27-31 p.

5. Ekim H, Özen S. Primary venous aneurysm of the external jugular vein. EJM. 2002;7:24-25.

6. Gordon DH, Rose JS, Kotimeir P, Levin DC. Jugular venous ectasia in children: a report of three cases and review of the literature. Radiology 1976;118:147-149. https://doi. org/10.1148/118.1.147
7. al Dousary S. Internal jugular phlebectasia. Int $J$ Pediatr Otorhinolaryngol 1997;38:273-280. https://doi.org/10.1016/So165-5876(96)01455-3

8. Reggina G, Rizzo S, Impedova G. Aneurysm of external jugular vein: case report and review of literature-a case report. Angiology. 1990;108:92-95.

9. Nwako FA, Agugua NE, Udeh CA, Osuorji RI. Jugular phlebectasia. $J$ Pediatr Surg. 1989;24:303-305. https://doi.org/10.1016/Soo 22-3468(89)80019-3

10. Indidharan R, Quah BS, Shuaib IL. Internal jugular phlebectasia-an unusual cause of neck swelling. Ann Trop Pediatr. 1999;19:105-108. https://doi.org/10.1080/02724939992725

11. Friedman SG, Krtishnasastry KV, Doscher W, et al. Primary venous aneurysms. Surgery. 1990;108:92-95. 